\title{
Monetary Trading: An Optimal Exchange System*
}

\author{
Guilherme Carmona \\ Universidade Nova de Lisboa
}

October 15, 2002

\begin{abstract}
We show that monetary trading is simple, self-enforcing, symmetric, and irreducible in a natural framework. Furthermore, we will show that the utility for each economic agent is at least as big under the monetary system as under any other simple, self-enforcing, symmetric, and irreducible trading system of the same complexity. Thus, we rationalize the monetary nature of real-world trade as being an efficient way to achieve those properties.

"The search for a means of exchange is almost as old as mankind." - The Economist, December 22nd 2001, page 87.
\end{abstract}

\section{Introduction}

Throughout time the means of exchange have changed considerably. Many commodities have been used as media of exchange, including chocolate, shells,

*This paper is an heavily revised version of Chapter 4 of my Ph.D. thesis. I wish to thank my advisors, Narayana Kocherlakota, and Marcel K. Richter, for their guidance, encouragement, and very helpful comments and suggestions. I wish also to thank Beth Allen, Pedro Amaral, Kemal Badur, Mehmet Barlo, Marco Basseto, Larry Gray, Leonid Hurwicz, Max Jodeit, Tim Kehoe, Patrick Leoni, Andy McLennan, Chris Phelan, Warren Weber, and Jan Werner, for very helpful comments. While they all improved the material in many ways, all remaining errors are mine. Financial support from the Subprograma Ciência e Tecnologia do $2^{\circ}$ Quadro Comunitário de Apoio is greatufully acknowledged. 
butter, salt, and, of course, paper. Recently, technological developments led to a substantial increase of cash substitutes like electronic payments and credit cards in most developed countries. ${ }^{1}$ Despite all the technological developments in payment systems, the nature of exchange in developed economies is strikingly similar to what is has been before - each person has a balance, which rises when he gives up goods, and falls when he acquires goods. The trading system is, in an informational sense, still a monetary one: all that has changed is that the balance, which was once physical, has become virtual.

This persistence of monetary exchange is puzzling. Given the advances in record-keeping and communication technology, it now seems feasible to design a trading system which conditions trading behavior in information that monetary exchange ignores. By taking into account that information, such a trading system has the potential to be more efficient than a monetary one. In fact, Kocherlakota and Wallace (1998) explicitly design a non-monetary trading system, which is more efficient than a monetary system whenever the record-keeping technology is sufficiently developed. Why do not real-world trading systems exploit these efficiency gains? Why do we observe monetary trading and not some other trading arrangement?

In this essay, we provide an answer to these questions. Several authors have pointed out that many real life institutions are self-enforcing, treat individuals symmetrically, cannot be simplified, and their rules are simple to understand. Furthermore, they argue that those properties seem necessary for any social institution to endure. Following their work, we will construct in a natural framework a monetary trading system that is simple, self-enforcing, symmetric, and irreducible. Furthermore, we will show that the utility for each economic agent is at least as big under the monetary system as under any other simple, self-enforcing, symmetric, and irreducible trading system of the same complexity. In other words, any trading system having the properties necessary to endure can be (weakly) dominated by a monetary trading system of the same level of complexity. In this sense, monetary trading is an optimal social institution, thus rationalizing the monetary nature of real-world trade.

We will describe our model by listing its time and uncertainty structure, its agents, the actions available to them, and their preferences over different action combinations; hence, we consider a noncooperative stochastic game

\footnotetext{
${ }^{1}$ Humphrey and Vesala (1996) found that "in all (fourteen) developed countries but the United States, electronic payments have been either the sole or the primary reason for the thirty four percent rise in total non-cash payments between 1987 and 1993."
} 
(Sobel (1971)) as a natural model of the type of society we are interested in. As is stressed by Wallace (1997), and is standard in monetary economics models, we will consider a society in which trade between its members is difficult. The nature of this difficulty is what is called absence of double coincidence of wants and consists of the following: when any pair of its members meet, it will never be the case that both have the goods the other would like to consume. ${ }^{2}$

Following the work of Aumann (1981), and Selten (1975), we will focus on strategies that can be played by finite automata, ${ }^{3}$ and that are subgame perfect. The reason we impose such restrictions is that those equilibria can be naturally classified as simple, and self-enforcing, respectively. We will also focus on automata that are symmetric in a similar way as in OkunoFujiwara and Postlewaite (1995), and are irreducible in the sense that any state of the automaton can be reached from any other state. Note that a reducible automaton can be simplified by reducing one state in a way that it produces the same outcome. Thus, only irreducible automata may be impossible to simplify. ${ }^{4}$ Finally, following Rubinstein (1986), the complexity of an automaton is defined to be the size of the state space.

We will formulate the notion of a monetary system as an automaton, where the state space will represent money holdings, the transition function will represent how money changes hands according to production and consumption actions, and the behavior function will represent a monetary behavior, in the sense that a seller produces for a buyer if and only if the buyer has enough money.

Our main results are that the monetary automaton is simple, subgameperfect, symmetric, and irreducible, and that the utility for each player is at least as big under the monetary automaton as under any other simple, subgame-perfect, symmetric, and irreducible automata of the same complex-

\footnotetext{
${ }^{2}$ The assumption that it will never be the case that both agents have the goods the other would like to consume is made only for simplicity. The same results would hold in a model where sometimes (but not always) the agents could exchange goods for goods.

${ }^{3}$ An automaton is described by a set of states (one of which is specified to be the initial state), by a transition function (which gives the next period's state as a function of the current period's state and actions), and by a behavior function (which prescribes behavior according to the state of the automaton). As Kalai and Stanford (1988) have shown, an automaton is an equivalent way of describing a strategy, and so throughout this paper the two terms will be used synonymously.

${ }^{4}$ Irreducibility is also a necessary condition for semi-perfection, an equilibrium concept developed by Rubinstein (1986).
} 
ity. It implies that our monetary system solves an institutional design problem - it is a Pareto efficient way of obtaining those properties. In this way we validate a long standing conjecture by Hurwicz (1980), revisited recently by Kocherlakota (1998b) and Wallace (2001), which rationalizes the monetary nature of real-world trade.

\section{Related Literature}

Although the focus of the present work is on monetary economics, one can pose the following broader question: can society design a simple set of rules in order to achieve certain goals? This question led Okuno-Fujiwara and Postlewaite (1995) to formalize the intuitive notion of a social norm and to study what would happen if the interactions between society members were directed by some social norm.

In particular, they consider a version of the infinitely repeated prisoners' dilemma with a continuum of players and random matching and they define in this setting a social norm as a special class of strategies. They then show that the Folk Theorem (Fudenberg and Maskin (1986)) holds even when we restrict attention to social norms rather than arbitrary strategies. In other words, given any individually rational payoff, there is a social norm with the property that it supports that payoff and that the actions it prescribes are optimal for every player given that the other players are using that social norm. Later on, Kandori (1992) extended their results to the finite players case, but the message was the same: the use of social norms may be enough to sustain any individually rational payoff as a sequential equilibrium payoff (provided that the discount factor is high enough), even in situations where the information that each of its members have about the others is limited.

The way those outcomes are supported as an equilibrium is by using a particular type of norm, in which an observable label is attached to each person and those labels completely determine players' behavior. More precisely, a person that deviates from the equilibrium path is labelled 'bad,' and nobody cooperates with a bad person; hence, nobody will cooperate with a person that has deviated before. Such a label may not be available in many circumstances, but as Ellison (1994), and Gata (1995) have shown efficient outcomes can still be sustained as an equilibrium outcome without the use of any explicit label. ${ }^{5}$

\footnotetext{
${ }^{5}$ Kandori (1992) also considers the case in which there is not an explicit labelling
} 
The above results suggest that those social norms could allow for a efficient level of trade in a trading model in which each player has limited information about the others. In Carmona (2000), we used a random matching model with finitely many players similar to both the Kiyotaki and Wright (1989) model, and the framework used by Ellison (1994), and we showed that indeed monetary trading is Pareto dominated by the Kandori-Ellison norm. Thus, we ask why the type of norm they studied is not used in most societies, and why do we observe monetary trading instead. ${ }^{6}$

A closely related line of research was explored by Townsend (1986). He considers several environments characterized by spatial separation, private information and a need to keep track of the past. He then formalizes and compares different types of communication-accounting systems, concluding that these systems can be ranked in terms of social welfare, from the worst to the best, as follows: oral assignment, portable object, written message and telecommunication systems. However, he compares those different types of communication-accounting systems assuming that all the agents in the economy will abide by the rules set by the social planner, except possibly regarding the revelation of private information; in particular, conditional on the information agents reveal, it is assumed that all agents will agree to the allocation chosen by the social planner. In others words, Townsend does not study how those different types of communication-accounting systems compare in a situation where the member of the society act strategically and in a decentralized way.

Our analysis differ also from that of Townsend (1986) in the following aspects: First, since we define a system to be a strategy, we abstract from many of its details; in particular, we abstract from any physical object which may be used in order to achieve the behavior prescribed by the institution. Second, we compare monetary behavior to all possible behavior, not only to a finite set of alternatives. Third, we obtain that a monetary system is an optimal payment arrangement in a given class of strategies, which is not the case in Townsend (1986) - of course, these discrepancies result from the different environments, and especially, from the different class of strategies we consider.

Similarly, Kocherlakota (2002), in an environment with absence of double

technology. In fact, Ellison (1994) uses the same strategy as in section 3 of Kandori (1992).

${ }^{6}$ Note that the norm studied by Kandori (1992) and Ellison (1994) seem to be used in some societies, namely in the archaic societies studied by Mauss (1990). 
coincidence of wants, presents a "portable-object system" that can implement frequent trade between society members. Although both his system and a monetary system are examples of portable objects systems, they are quite different. Hence, this paper does not address the question of why most societies use a monetary system.

A monetary system is a possible way to allow for frequent trade between society members. However, as discussed above, there are other ways of accomplishing frequent transactions, namely the communication-accounting systems described by Townsend (1986), the portable object system described by Kocherlakota (2002) and the social norms studied by Kandori (1992), Ellison (1994) and Okuno-Fujiwara and Postlewaite (1995).

These remarks lead us to Hurwicz (1980)'s conjecture that money might be an efficient way to solve some institutional design problem, which we validate by showing that monetary trading is a Pareto efficient way of obtaining certain properties typically associated with social institutions. The importance of this result is that it allow us to regard money not only as an equilibrium outcome, but also, and more importantly, as an optimal equilibrium.

We note, however, that our optimality result for monetary trading is not the only one available. It is well know that in overlapping generations models, the monetary equilibrium can be Pareto optimal (see Samuelson (1958).) Also, Kocherlakota and Wallace (1998) show that in a random matching model monetary trading is the optimal trading arrangement when each person's actions is private information to the person.

What causes our result to differ from those above is that the properties that we use to rationalize monetary trading are typically associated with social institutions. The view we express in this paper, which is substantially different from the above, is that monetary trading satisfies in optimal way the properties that any exchange system, as a particular case of a social institution, need to endure.

Furthermore, as we mentioned in the introduction, our result allow us to explain why real-world trading systems still have a monetary nature despite the recent advances in record-keeping and communication technology. This is in contrast with Kocherlakota and Wallace (1998) which explicitly design a non-monetary trading system that will be more efficient than a monetary system whenever the record-keeping technology is sufficiently developed. 


\section{The Basic Model}

There are two people, and they meet in every period $t \in \mathbb{N}$. In every period, a given person can be a producer or a consumer; we will assume that will be decided by nature, in a way that each person will be the producer with $1 / 2$ probability.

In our economy consumers and producers are different in the following way: only the producer has to make a choice, which is whether to produce (for the consumer) or not. If the producer chooses to produce, he suffer a utility cost $d>0$, and the consumer receives a positive utility $u>0$. If the producer chooses not to produce, them both himself and the consumer will receive zero utility.

We will assume that production is efficient in the sense that the benefit derived by the consumer exceeds the cost incurred by the producer. That is, we will assume that $u>d$.

Let $N=\{1,2\}$ stand for the set of players, $\Omega=\{1,2\}$ for the set of states of nature, and $A=\{P, N P\}$ for the set of possible actions. We make the convention that when the state of nature equals 1 , then player 1 is the producer, and he will choose an action from the set $A$; similarly, when the state of nature equals 2 , then player 2 is the producer. The payoff players receive period-wise, which depend on the state of the nature and on the choice made by the producer, are summarized in the following table:

\begin{tabular}{|c|c|c|}
\hline$\omega$ & $\mathrm{P}$ & $\mathrm{NP}$ \\
\hline \hline 1 & $-d, u$ & 0,0 \\
2 & $u,-d$ & 0,0 \\
\hline
\end{tabular}

We denote the period-wise payoffs as $u_{i}(\omega, a)$.

Intuitively, these state-of-nature-dependent payoffs try to capture a two person trade meeting, in which there is absence of double coincidence of wants. We should note that the model is not to be taken literally; in fact, it is designed to capture the essence of the standard random-matching models of money (Kiyotaki and Wright (1989)) in a simple way.

In the standard random matching models of money, the essential feature is that every agent, in every period of time, has a $\frac{1}{3}$ probability of meeting an agent who likes the good he produces but who can not produce the good he likes (i.e., he has a $\frac{1}{3}$ probability of being a seller, in the above sense); has 
a $\frac{1}{3}$ probability of meeting an agent who does not like the good he produces but who can produce the good he likes (i.e., he has a $\frac{1}{3}$ probability of being a buyer, in the above sense); and finally, has a $\frac{1}{3}$ probability of meeting an agent who neither likes the good he produces nor can produce the good he likes (in our model, there is no situation that corresponds to this one; however, it could be added without changing the results).

Furthermore, our model belongs to the class of absence of double coincidence models of money. Wallace (1997) defines the general class of absence of double coincidence models of money in terms of four properties: first, any such model "contains more than one time period"; second, it "has two-person meetings"; third, "a specialization pattern among people motivates trade and is consistent with the lack of double coincidences in two-person meetings"; and fourth, "something in the model prevents trade from being accomplished through some prior arrangement or through the use of some form of credit." Clearly, our model satisfies the first three properties. Moreover, the assumptions of Theorem 1 below will prevent trade from being accomplished through the use of some form of credit. ${ }^{7}$

The interaction described above takes place in every period $t \in \mathbb{N}$. Each player's payoff in the repeated game depends on the payoff he receives in all periods, in the following way: if $\boldsymbol{\omega}=\left\{\omega_{k}\right\}_{k=1}^{\infty} \subseteq \Omega$ is a sequence of states of nature and if $\mathbf{a}=\left\{a_{k}\right\}_{k=1}^{\infty} \subseteq A$ is a sequence of actions, then player $i$ 's payoff equals

$$
U_{i}(\boldsymbol{\omega}, \mathbf{a})=\limsup _{n \rightarrow \infty} \frac{1}{n} \sum_{k=1}^{n} u_{i}\left(\omega_{k}, a_{k}\right) .
$$

By using the above payoff function, the payoff of any finite automaton, which will be described in the following section, equals the limit, as the discount factor goes to one, of the payoffs computed using the discounted sum criterion. Since in a monetary system players will produce in exchange for the future benefits of having an extra amount of money, our results would only hold in the discounted case for a sufficiently high discount factor that is, in the limit case. Thus, the above payoff function allow us to present our results in a clearer way, and also, it allows us to simplify some of their proofs.

\footnotetext{
${ }^{7}$ This is actually a delicate point. See section 7 for a more detailed discussion.
} 


\section{Automata, and Monetary Trading}

We will describe the behavior of each player by an automaton. An automaton for player $i$ is a triple $I_{i}=\left(\left(S_{i}, \bar{s}_{i}\right), T_{i}, B_{i}\right)$ where: $S_{i}$ is a set of states; $\bar{s}_{i} \in S_{i}$ is the initial state; $T_{i}: \Omega \times S_{i} \times A \rightarrow S_{i}$ is a transition function; and $B_{i}: S_{i} \rightarrow A$ is a behavior function.

A pair of individual automata $I=\left(I_{1}, I_{2}\right)$, or for short, an automaton, together with a sequence of states of nature $\boldsymbol{\omega}=\left\{\omega_{k}\right\}_{k=1}^{\infty} \subseteq \Omega$ induce a sequence of actions $\mathbf{a}(I, \boldsymbol{\omega})=\left\{a_{k}\right\}_{k=1}^{\infty} \subseteq A$ in the following way: $a_{1}=$ $B_{\omega_{1}}\left(\bar{s}_{\omega_{1}}\right)$, and $a_{k}=B_{\omega_{k}}\left(s_{\omega_{k}}^{k}\right)$, where $s_{i}^{k}=\bar{T}_{i}\left(s_{i}^{k-1}, a_{k-1}\right)$, for both $i=1,2 .^{8}$

An automaton, together with a sequence of realizations of the uncertainty, induce a sequence of actions. Since we can compute the payoff generated by a sequence of realizations of the uncertainty, and actions, we can compute the payoff of an automaton $I$ as follows: first, for $i=1,2$, we define a function $\boldsymbol{\omega} \mapsto U_{i}(I)(\boldsymbol{\omega})$ by defining

$$
U_{i}(I)(\boldsymbol{\omega})=U_{i}(\boldsymbol{\omega}, \mathbf{a}(I, \boldsymbol{\omega})) .
$$

Then, payoff of an automaton $I$ for player $i, i=1,2$, is

$$
U_{i}(I)=\int_{\Omega} U_{i}(I)(\boldsymbol{\omega}) d \mu
$$

where $\Omega=\Omega \times \Omega \times \cdots$, and $\mu$ is the usual product measure.

One of the reason we choose to describe players behavior using automata is because it allows to describe monetary trading in a very convenient way. Its definition tries to capture the way people trade in actual monetized societies, in which one person gives goods and receives money, while some other gives money and receives goods.

A monetary system $I_{M}=\left(I_{1}^{M}, I_{2}^{M}\right)$ with $M \in \mathbb{N}$ units of money and initial money holdings $\bar{s}_{M}$ will be described as follows: the set of states is

$$
S_{1}^{M}=S_{2}^{M}=S_{M}=\{0, \ldots, M\},
$$

and the initial state is $\bar{s}_{M}$. The transition function $T_{1}^{M}=T_{2}^{M}=T_{M}$ :

\footnotetext{
${ }^{8}$ Recall that player $i$ is the producer in period $k$ if $\omega_{k}=i, i=1,2$.
} 
$\Omega \times A \times S_{M} \rightarrow S_{M}$ is defined by:

$$
\begin{aligned}
T_{M}(1, P, m) & = \begin{cases}m+1 & \text { if } m \leq M-1, \\
m & \text { otherwise, }\end{cases} \\
T_{M}(2, P, m) & = \begin{cases}m-1 & \text { if } m \geq 1, \\
m & \text { otherwise }\end{cases} \\
T_{M}(\omega, N P, m) & =m,
\end{aligned}
$$

The interpretation is as follows: $S_{M}$ represents the set of possible money holdings for player 1; clearly, we could obtain a similar definition for a monetary system if the set of status levels were $\left\{\left(m_{1}, m_{2}\right) \in\{0, \ldots, M\} \times\right.$ $\left.\{0, \ldots, M\}: m_{1}+m_{2}=M\right\}$. Regarding $T_{M}$, the intuition is: when player 1 is the seller, that is, when $\omega=1$, if he produces he receives one additional unit of money, unless player 2 does not have any money. When player 1 is the buyer, that is, when $\omega=2$, if player 2 produces for him, he has to give one unit of money to player 2 , unless he (player 1) does not have any money. In the remaining cases, there is no change in the amount of money player 1 has.

Player 1's behavior function is defined as follows:

$$
B_{1}^{M}(m)=\left\{\begin{array}{l}
P \text { if } m<M, \\
N P \text { otherwise }
\end{array}\right.
$$

Similarly, Player 2's behavior function is defined as follows:

$$
B_{2}^{M}(m)=\left\{\begin{array}{l}
P \text { if } m>0, \\
N P \text { otherwise; }
\end{array}\right.
$$

Intuitively, any player produces if and only if he is a seller and the other player has a positive amount of money. The definition of $S_{M}, T_{M}$ and $B_{M}$ describes a "monetary behavior" in the sense that in a two people meeting one person gives goods and receives money, while the other gives money and receives goods; further, it seems to be the only natural way of describing such a monetary behavior.

Note that the monetary automaton satisfies many symmetry properties. First, we have that players use a common state space, initial state, and transition function: $S_{1}^{M}=S_{2}^{M}, T_{1}^{M}=T_{2}^{M}$, and $\bar{s}_{1}^{M}=\bar{s}_{2}^{M}$. Second, some states can be associated in a natural way: if we define $\phi(m)=M-m$, we obtain a bijection $\phi: S^{M} \rightarrow S^{M}$, satisfying $B_{1}^{M}(m)=B_{2}^{M}(\phi(m))$. Third, 
the monetary automaton induces a Markov chain on $S^{M}$, described by a symmetric transition matrix. In fact, if $\Pi_{M}$ denotes such matrix, one easily sees that the nonzero entries of $\Pi_{M}$ are:

$$
\begin{aligned}
\pi_{0,0} & =\frac{1}{2}, \pi_{0,1}=\frac{1}{2} \\
\pi_{m, m-1} & =\frac{1}{2}, \pi_{m, m+1}=\frac{1}{2}, \text { for all } 0<m<M \\
\pi_{M, M-1} & =\frac{1}{2}, \pi_{M, M}=\frac{1}{2} .
\end{aligned}
$$

Generalizing from the particular case of the monetary automaton, we say that an automaton $I=\left(I_{1}, I_{2}\right)$ is symmetric if: (1) $S_{1}=S_{2}, T_{1}=T_{2}$, and $\bar{s}_{1}=\bar{s}_{2} ;(2)$ there exist a bijection $\phi: S \rightarrow S$ such that $B_{1}(s)=$ $B_{2}(\phi(s))$; and (3) $I$ induces a Markov chain on $S$, described by a symmetric transition matrix. ${ }^{9}$ Intuitively, the class of symmetric automata consist of those that in which different individuals in the same situation determined by the realization of the uncertainty and with the same state are prescribed the same action. ${ }^{10}$

\section{Monetary Trading as an Optimal Social In- stitution}

In our model the two players interact in every period of time. This interaction is described by an automaton $I$, which consist of a pair of individual automata: $I=\left(I_{1}, I_{2}\right)$. By changing each player's automaton, we obtain different outcomes, some of which may be unreasonable.

The first requirement we impose on the automaton players use is that it is self-enforcing. More precisely, we will require that each player, given

\footnotetext{
${ }^{9}$ Note that any finite automaton $I$ induces a Markov chain defined through by the following transition matrix $\Pi$ :

$$
\pi_{s s^{\prime}}= \begin{cases}1 & \text { if } T\left(\omega, s, B_{\omega}(s)\right)=s^{\prime} \text { for all } \omega=1,2 \\ 0 & \text { if } T\left(\omega, s, B_{\omega}(s)\right) \neq s^{\prime} \text { for all } \omega=1,2 \\ \frac{1}{2} & \text { otherwise }\end{cases}
$$

${ }^{10}$ Condition 3 in the definition of a symmetric automaton is far less intuitive. It would be interesting to dispense with that condition, but unfortunately we have not been able to do so.
} 
the other player behavior, have an incentive to act the way as the social institution prescribes at all possible contingencies. Formally, this amounts to require that the automaton is a subgame perfect equilibrium.

Before giving the formal definition, we need the following notation: given an automaton $I=((S, \bar{s}), T, B)$, then $(I, s)$ denotes the automaton $((S, s), T, B)$; that is, $(I, s)$ differs from $I$ only on the initial state. We then say that an automaton $I$ is a subgame perfect equilibrium if for all $i=1,2, s$ in $S=S_{1} \times S_{2}$, and any player $i$ 's automaton $I_{i}^{\prime}$, we have that

$$
U_{i}(I, s) \geq U_{i}\left(\left(I_{i}^{\prime}, I_{-i}\right),\left(s^{\prime}, s_{-i}\right)\right)
$$

A second requirement we impose is that there are no obsolete states: all states should be used regularly in the regular course of the game. As Rubinstein pointed out "[these] considerations have some similarity to phenomena frequently observed in real life: social institutions, various types of organizations, and human abilities degenerate or are readily discarded if they are not used regularly." Formally, we say that a symmetric automaton I is irreducible if the Markov chain induced by $I$ is irreducible. ${ }^{11}$

The view that we take here is that, in our framework, only automata that are finite, symmetric, subgame perfect, and irreducible can describe a social institution. For $N \in \mathbb{N}$, let $\mathcal{A}_{N}$ be the set of all symmetric, irreducible, subgame perfect automata with a state space having no more than $N$ elements. Our main result is:

Theorem 1 For $N \in \mathbb{N}, I_{M}$, with $M=N-1$, solves

$$
\max _{I \in \mathcal{A}_{N}} U_{1}(I)+U_{2}(I)
$$

Theorem 1 asserts that not only the monetary automaton is a symmetric, irreducible, subgame perfect automaton for any possible amount of money, but in fact, it is efficient within that class. In particular, any finite, symmetric, irreducible, subgame perfect automaton can be (weakly) dominated by the monetary trading system of the same level of complexity. In this sense, monetary trading is an optimal social institution.

\footnotetext{
${ }^{11}$ A Markov chain represented by a transition matrix $\Pi$ is irreducible if for all states $s$, and $s^{\prime}$ there exists $K \in \mathbb{N}$ such that $\pi_{s, s^{\prime}}^{(K)}>0$.
} 


\section{On the Proof of Theorem 1}

Although we have to deal with some technical detail to prove Theorem 1, the ideas behind it are simple. In this section we will thus present the main ideas, and we leave for the appendix a detailed proof.

The first step of the proof is to show that any monetary automaton is symmetric, irreducible, and subgame perfect. One easily sees that any monetary automaton is symmetric. Since any state can lead to the two adjacent states, with the convention that state 0 and state $M$ each is adjacent of itself, one can conclude that any monetary automaton is irreducible.

To show that each monetary automaton $I_{M}$ is a subgame perfect equilibrium is more delicate. We first study the case in which each player $i$ payoff function in the repeated game equals

$$
U_{i}^{\delta}(\boldsymbol{\omega}, \mathbf{a})=(1-\delta) \sum_{k=1}^{\infty} \delta^{k} u_{i}\left(\omega_{k}, a_{k}\right),
$$

and then we take the limit of $U_{i}^{\delta}$ with $\delta$ converging to 1 ; existing results guarantee that if $I_{M}$ is subgame perfect for all discount factors close to 1 , then $I_{M}$ is subgame perfect in our game.

It is useful to use the discounted version of our game to show that $I_{M}$ is subgame perfect because in discounted games we can use the one-shot deviation principle (see Abreu (1988).) For the particular case of a monetary automaton, we need to show that it is not profitable for a producer to refuse to produce for a consumer with money, and follow the monetary strategy afterwards. If a producer deviates by not producing to a consumer with money, his utility increases today by $d$, i.e., he gains by not having to produce. However, he starts next period with one less unit of money, and the other player starts with one more unit of money. Hence, the difference of payoffs is

$$
\begin{aligned}
& (1-\delta) d+\delta\left[U_{i}^{\delta}\left(I_{M}, m\right)-U_{i}^{\delta}\left(I_{M}, m+1\right)\right]= \\
& (1-\delta)\left[d+\frac{\delta}{1-\delta}\left(U_{i}^{\delta}\left(I_{M}, m\right)-U_{i}^{\delta}\left(I_{M}, m+1\right)\right)\right] .
\end{aligned}
$$

Thus, to show that a deviation is not profitable for all discount factors close to 1 , it is enough to show that

$$
\lim _{\delta \rightarrow 1} \frac{1}{1-\delta}\left[U_{i}^{\delta}\left(I_{M}, m+1\right)-U_{i}^{\delta}\left(I_{M}, m\right)\right]>d,
$$


that is, the next step is to estimate the value of starting the game with one extra unit of money.

If a given player $i$ starts with an extra unit of money, it means that the other player starts with one less unit of money. One can conjecture that either player $i$ will consume one more time by starting with one extra unit of money, or she will produce one less time. This conjecture turns out to be true, and in fact one obtains

$$
\lim _{\delta \rightarrow 1} \frac{1}{1-\delta}\left[U_{i}^{\delta}\left(I_{M}, m+1\right)-U_{i}^{\delta}\left(I_{M}, m\right)\right]=\alpha_{m} u+\left(1-\alpha_{m}\right) d
$$

where $0<\alpha_{m}<1$ is the probability that player $i$ will consume one more time by starting with $m+1$ units of money instead of starting with $m$ units of money. Since by assumption $u>d$, this result implies that $I_{M}$ is a subgame perfect equilibrium for all $\delta$ close enough to 1 .

The second step of the proof is to show that $U_{i}\left(I_{M}\right) \geq U_{i}(I)$ for any $i=1,2$, and any symmetric, irreducible, and subgame perfect automaton $I$ with $|S| \leq\left|S_{M}\right|$. Using standard ergodic theorems for Markov chains, one sees that

$$
U_{i}(I)=\frac{1}{2|S|} \sum_{s \in S} \sum_{\omega \in \Omega} u_{i}(\omega, B(s)) ;
$$

hence, in particular,

$$
\begin{aligned}
U_{i}\left(I_{M}\right) & =\frac{1}{2(M+1)}[-d+\underbrace{(u-d)+\cdots+(u-d)}_{M-1 \text { terms }}+u] \\
& =\frac{u-d}{2}\left(1-\frac{1}{M+1}\right) .
\end{aligned}
$$

Note that in a symmetric automata the number of states in which player 1 produces equals the number of states in which player 2 produces. Since production is efficient (i.e., since $u>d$,) the best symmetric outcome is obtained when production takes place in every state. The above expression for $U_{i}\left(I_{M}\right)$ says that in the monetary automaton production occurs in all but one state. More precisely, for every $\omega$ there is only one state in which production does not take place, which is when the consumer does not have money. Since in every subgame perfect equilibrium there has to be a state in which a given player does not produces - otherwise the strategy of always producing would be an equilibrium, which one can easily see not to be the 
case - it follows that each player's utility of any symmetric, irreducible, and subgame perfect automaton is at most

$$
\frac{u-d}{2}\left(1-\frac{1}{|S|}\right) .
$$

This concludes the argument, since $I$ is assumed to have at most as much states as the monetary automaton, and so

$$
1-\frac{1}{|S|} \leq 1-\frac{1}{\left|S_{M}\right|}=1-\frac{1}{M+1}
$$

\section{Concluding Remarks}

Our main result provides a rationale for the monetary nature of exchange. We rationalized the monetary nature of exchange by showing that in a class of economies a monetary system can be understood as a best strategy among a particular class of strategies.

More precisely, we have shown that there exists a class $\mathcal{E}_{0}$ of economies (in our case indexed by the parameters $u$, and $d$ ) such that for all possible money supplies $M$, and initial money holdings $m_{0}$ there exist a monetary system $I_{M, m_{0}}$, and a class of automata $\mathcal{I}_{0}$ such that $I_{M, m_{0}}$ is Pareto optimal in $\mathcal{I}_{0}$.

The first remark we make is on what is meant by a monetary system. As we defined it in section 4 , the essential features of it is that each player conditions his behavior on money holdings in a way that he produce if and only if his trading partner has money. Furthermore, the amount of money that a given player has increases one unit when he produces, and decreases one unit when he consumes (except possibly when the consumer has no money, or when the producer has all the money in the economy.)

Implicitly, in our definition of a monetary system, we are assuming that the price level equals 1 in every period. The stationarity of the price level does play a role in our optimality result. In fact, it may remind us of standard results from overlapping generations models, which state that the stationary monetary equilibrium with constant price level is Pareto optimal provided that (and because) the total amount of resources is also stationary (see, for example, Champ and Freeman (1994).) As the following example illustrates, a similar phenomenon takes place in our economy. 
The following automaton $I_{M}^{2}$ describes a monetary system in which the price level is 1 in odd periods, and 2 in even periods. We assume that there are $M$ units of money in the economy, with $M$ being an even natural number. The transition function, and the behavior function for each player is analogous to the monetary automaton of section 4 in odd periods, and differs from this in even period since trade is conducted at an higher price (a precise description of $I_{M}^{2}$ is given in Appendix B.) We can show that, for $i=1,2$,

$$
\begin{aligned}
U_{i}\left(I_{M}^{2}\right) & =\frac{u-d}{2}\left(1-\frac{3}{2(M+1)}\right) \\
& <\frac{u-d}{2}\left(1-\frac{1}{M+1}\right)=U_{i}\left(I_{M}\right) .
\end{aligned}
$$

Thus, a non-stationary price level leads to a drop in utility for all players. This happens because trade is less frequent in those periods in which the price is high. Also, note that the complexity (i.e., the number of states) of the monetary automaton with constant prices is $M+1$, while the complexity of the automaton $I_{M}^{2}$ is $2(M+1)$ - when prices are changing, players need to keep track of more information. However, a monetary automaton with constant prices, ${ }^{12}$ and $2(M+1)$ states yields an even higher payoff. Thus, non-stationary prices are non-optimal because trade is less frequent, and, more complex.

We emphasize that our main result rationalizes the monetary nature of trade. When we defined monetary trading, although we have referred to the states of that particular automaton as money, we did not commit ourselves to any particular type of money. As we pointed out in the introduction, money in our model can be made of chocolate, gold, or paper; in fact, it can be simply memory as in Kocherlakota (1998a) — all these alternatives produce the same outcome.

An alternative that in our model is equivalent to monetary trading is private credit, provided that there is a limit to the amount of debt each player can have. This is, of course, a unpleasant feature of our model. However, in a model with more than two players, one would be able to distinguish money

\footnotetext{
${ }^{12}$ Note that assuming that the price is equal to 1 is without loss of generality: a monetary system with price equal to 2 , total money equal to 5 , and initial money holdings of player 1 equal to 3 , is equivalent to a monetary system with price equal to 1 , total money equal to 3 , and initial money holdings of player 1 equal to 2 .
} 
from credit: monetary trading involves only one balance per person, while private credit requires a balance for every pair of players. Furthermore, we suspect that our main result extent to the multi-player model of Carmona (2000), which would rationalize monetary exchange in a framework that allow us to explicitly distinguish money from credit. ${ }^{13}$

The interpretation that our main result provides a class of economies, and a class of strategies in which monetary trading is optimal, suggests additional generalizations: progress in the question of why do we observe monetary trading can be obtained by similar results in a larger class of economies, and which consider larger classes of strategies.

\section{A Proof of Theorem 1}

In this appendix, we prove Theorem 1. We will start by showing that if for each player $i$, his payoff in the repeated game equals

$$
U_{i}^{\delta}(\boldsymbol{\omega}, \mathbf{a})=(1-\delta) \sum_{k=1}^{\infty} \delta^{k} u_{i}\left(\omega_{k}, a_{k}\right)
$$

then the monetary automaton is a subgame perfect equilibrium.

The following lemma estimates the benefit for a given player of having one extra unit of money.

Lemma 1 Let $m \in\{0, \ldots, M-1\}$. Then there is $\alpha_{m} \in(0,1)$ such that

$$
\lim _{\delta \rightarrow 1} \frac{1}{1-\delta}\left(U_{i}^{\delta}\left(I_{M}, m+1\right)-U_{i}^{\delta}\left(I_{M}, m\right)\right)=\alpha_{m} u+\left(1-\alpha_{m}\right) d,
$$

for $i=1,2$.

Moreover, for all $\delta \in(0,1)$, and $i=1,2$,

$$
\frac{1}{1-\delta}\left(U_{i}^{\delta}\left(I_{M}, m+1\right)-U_{i}^{\delta}\left(I_{M}, m\right)\right) \leq \alpha_{m} u+\left(1-\alpha_{m}\right) d
$$

Proof. Because player 1's case is symmetric to player 2's, we deal only with the former.

Step 1: Some definitions.

\footnotetext{
${ }^{13}$ What prevent us to generalize our main result to the multi-player case is that we lack an analogue of Lemma 1.
} 
Denote $m(0)=(m+1, M-m-1)$ and $m^{\prime}(0)=(m, M-m)$. Also, let $\Omega:=\Omega \times \Omega \times \ldots$ be the countable infinite Cartesian product of $\Omega$, and let $(\Omega, \mathcal{G}, \mu)$ denote the usual corresponding probability space. A generic element of $\boldsymbol{\Omega}$ is denoted by $\boldsymbol{\omega}=\left\{\omega_{t}\right\}_{t=1}^{\infty}$, where $\omega_{t} \in \Omega$, for all $t \in \mathbb{N}$. Given $\boldsymbol{\omega}$, let $m(k)(\boldsymbol{\omega})=\left(m_{1}(k)(\boldsymbol{\omega}), m_{2}(k)(\boldsymbol{\omega})\right)$ denote the amount of money players have at the end of stage $k$ if they started with $m(0)$ and $m^{\prime}(k)(\boldsymbol{\omega})$ denote the amount of money players have at the end of stage $k$ if they started with $m^{\prime}(0)$.

With this notation, we can write

$$
\begin{aligned}
& \frac{1}{1-\delta}\left(U_{1}^{\delta}\left(I_{M}, m+1\right)-U_{1}^{\delta}\left(I_{M}, m\right)\right)= \\
& \frac{1}{1-\delta}\left(\int_{\Omega} U_{1}^{\delta}\left(I_{M}, m+1\right)(\boldsymbol{\omega}) d \mu-\int_{\Omega} U_{1}^{\delta}\left(I_{M}, m\right)(\boldsymbol{\omega}) d \mu\right)= \\
& \frac{1}{1-\delta}\left(\int_{\Omega}\left[U_{1}^{\delta}\left(I_{M}, m+1,\right)(\boldsymbol{\omega})-U_{1}^{\delta}\left(I_{M}, m\right)(\boldsymbol{\omega})\right] d \mu\right),
\end{aligned}
$$

where the last equality follows because both the functions $\boldsymbol{\omega} \mapsto U_{1}^{\delta}\left(I_{M}, m\right)(\boldsymbol{\omega})$ and $\boldsymbol{\omega} \mapsto U_{1}^{\delta}\left(I_{M}, m+1\right)(\boldsymbol{\omega})$ are integrable.

Step 2: There exists $\left\{A_{t}, B_{t}\right\}_{t=1}^{\infty}$ such that $\frac{1}{1-\delta}\left(U_{1}^{\delta}\left(I_{M}, m+1\right)-U_{1}^{\delta}\left(I_{M}, m,\right)\right)=$ $\sum_{t=1}^{\infty} d \delta^{t-1} \mu\left(B_{t}\right)+\sum_{t=1}^{\infty} u \delta^{t-1} \mu\left(A_{t}\right)$.

Let $A_{1}:=\left\{\boldsymbol{\omega} \in \boldsymbol{\Omega}: m_{1}(1)(\boldsymbol{\omega})=0\right.$ and $\left.\omega_{1}=2\right\}$; in $A_{1}$ player 1 is able to consume under $m$ but not in $m^{\prime}$. Note also that $m(1)=m^{\prime}(1)$. So, for $\boldsymbol{\omega} \in A_{1}$, the difference in payoffs is $u$. That is, for $\boldsymbol{\omega} \in A_{1}$,

$$
U_{1}^{\delta}\left(I_{M}, m+1\right)(\boldsymbol{\omega})-U_{1}^{\delta}\left(I_{M}, m\right)(\boldsymbol{\omega})=(1-\delta) u .
$$

Let $B_{1}:=\left\{\boldsymbol{\omega} \in \Omega: m_{2}^{\prime}(1)(\boldsymbol{\omega})=0\right.$ and $\left.\omega_{1}=1\right\}$; in $B_{1}$ player $i$ has to produce under $m^{\prime}$ but not under $m$. Note also that $m(1)=m^{\prime}(1)$. Thus, for $\boldsymbol{\omega} \in B_{1}$,

$$
U_{1}^{\delta}\left(I_{M}, m+1\right)(\boldsymbol{\omega})-U_{1}^{\delta}\left(I_{M}, m\right)(\boldsymbol{\omega})=(1-\delta) d .
$$

We proceed by induction: let $t \geq 2$. Let

$$
\left.A_{t}:=\left\{\boldsymbol{\omega} \in \boldsymbol{\Omega} \backslash\left(\left(\bigcup_{k=1}^{t-1} A_{k}\right) \cup\left(\bigcup_{k=1}^{t-1} B_{k}\right)\right): m_{1}(t)(\boldsymbol{\omega})=0 \text { and } \omega_{t}=2\right)\right\}
$$

and

$$
B_{t}=\left\{\boldsymbol{\omega} \in \boldsymbol{\Omega} \backslash\left(\left(\bigcup_{k=1}^{t-1} A_{k}\right) \cup\left(\bigcup_{k=1}^{t-1} B_{k}\right)\right): m_{2}^{\prime}(t)(\boldsymbol{\omega})=0 \text { and } \omega_{t}=1\right\} .
$$


Similarly as before, we have that for $\boldsymbol{\omega} \in A_{t}$,

$$
U_{1}^{\delta}\left(I_{M}, m+1\right)(\boldsymbol{\omega})-U_{1}^{\delta}\left(I_{M}, m\right)(\boldsymbol{\omega})=(1-\delta) \delta^{t-1} u,
$$

and for $\boldsymbol{\omega} \in B_{t}$,

$$
U_{1}^{\delta}\left(I_{M}, m+1\right)(\boldsymbol{\omega})-U_{1}^{\delta}\left(I_{M}, m\right)(\boldsymbol{\omega})=(1-\delta) \delta^{t-1} d .
$$

Finally let $C=\Omega \backslash\left[\left(\bigcup_{t=1}^{\infty} A_{t}\right) \cup\left(\bigcup_{t=1}^{\infty} B_{t}\right)\right]$.

For each $t \in \mathbb{N}, A_{t}$ is measurable since it can be written as $D_{1} \times \ldots \times$ $D_{t} \times \Omega \times \Omega \times \ldots$ for some $D_{1}, \ldots, D_{t} \in \Omega$. Similarly, $B_{t}$ is measurable for each $t \in \mathbb{N}$ and so is $C$. Note also that for all $j, k \in \mathbb{N}$, we have that $A_{j} \cap A_{k}=\emptyset$, $B_{j} \cap B_{k}=\emptyset$ and $A_{j} \cap B_{k}=\emptyset$.

Claim $1 \mu(C)=0$.

Proof. Let $S_{n}(\boldsymbol{\omega})$ be the number of times that $\omega_{k}=1$ in the first $n$ periods. If $\boldsymbol{\omega} \in C$, then it follows that $m_{1}(k)(\boldsymbol{\omega})>0$ whenever $\omega_{k}=2$, for all $k$. Therefore, $n-S_{n}$, which equals the number of times that $\omega_{t}=2$, is also the amount of money player 1 spent in the first $n$ periods. Since the amount of money player 1 receives in the first $n$ periods is at most $S_{n}$, then for each $n \in \mathbb{N}, m_{1}(0)+S_{n} \geq n-S_{n}$, that is $S_{n} / n \geq 1 / 2-m_{1}(0) / 2 n$. Hence $C \subseteq \bigcap_{n=1}^{\infty}\left\{\boldsymbol{\omega} \in \Omega: \frac{S_{n}(\boldsymbol{\omega})}{n} \geq \frac{1}{2}-\frac{m_{1}(0)}{2 n}\right\}$ which has measure zero by lemma 2 applied to the sequence of random variables $\left\{X_{n}\right\}_{n=1}^{\infty}$, where for all $n, X_{n}(\boldsymbol{\omega})=\chi_{\left\{\boldsymbol{\omega}: \omega_{n}=1\right\}}$.

Hence, we obtain

$$
\begin{aligned}
& \frac{1}{1-\delta}\left(U_{1}^{\delta}\left(I_{M}, m+1\right)-U_{1}^{\delta}\left(I_{M}, m\right)\right)= \\
& \frac{1}{1-\delta}\left(\sum_{t=1}^{\infty} \int_{A_{t}}\left[U_{1}^{\delta}\left(I_{M}, m+1\right)(\boldsymbol{\omega})-U_{1}^{\delta}\left(I_{M}, m\right)(\boldsymbol{\omega})\right] d \mu+\right. \\
& \left.+\sum_{t=1}^{\infty} \int_{B_{t}}\left[U_{1}^{\delta}\left(I_{M}, m+1\right)(\boldsymbol{\omega})-U_{1}^{\delta}\left(I_{M}, m\right)(\boldsymbol{\omega})\right] d \mu\right)= \\
& \sum_{t=1}^{\infty} u \delta^{t-1} \mu\left(A_{t}\right)+\sum_{t=1}^{\infty} d \delta^{t-1} \mu\left(B_{t}\right) .
\end{aligned}
$$

Step 3: There exists $\alpha_{m} \in(0,1)$ such that

$$
\lim _{\delta \rightarrow 1} \frac{1}{1-\delta}\left(U_{1}^{\delta}\left(I_{M}, m+1\right)-U_{1}^{\delta}\left(I_{M}, m\right)\right)=\alpha_{m} u+\left(1-\alpha_{m}\right) d .
$$


By Abel's theorem (DePree and Swartz (1988), Chapter 11, Theorem 17, page 135 ),

$$
\lim _{\delta \rightarrow 1} \frac{1}{1-\delta}\left(U_{1}^{\delta}\left(I_{M}, m+1\right)-U_{1}^{\delta}\left(I_{M}, m\right)\right)=d \mu\left(\bigcup_{t=1}^{\infty} B_{t}\right)+u \mu\left(\bigcup_{t=1}^{\infty} A_{t}\right) .
$$

So define $\alpha_{m}:=\mu\left(\bigcup_{t=1}^{\infty} A_{t}\right)$. Finally note that $\mu\left(\bigcup_{t=1}^{\infty} A_{t}\right) \geq\left(\frac{1}{2}\right)^{m(0)}>0, \mu\left(\bigcup_{t=1}^{\infty} B_{t}\right) \geq$ $\left(\frac{1}{2}\right)^{M-m(0)},\left(\bigcup_{t=1}^{\infty} A_{t}\right) \cap\left(\bigcup_{t=1}^{\infty} B_{t}\right)=\emptyset$ and that $\Omega=\left(\bigcup_{t=1}^{\infty} A_{t}\right) \cup\left(\bigcup_{t=1}^{\infty} B_{t}\right) \cup C$ implying that $\mu\left(\bigcup_{t=1}^{\infty} A_{t}\right)+\mu\left(\bigcup_{t=1}^{\infty} B_{t}\right)=1$.

Step 4: For all $\delta \in(0,1)$,

$$
\frac{1}{1-\delta}\left(U_{1}^{\delta}\left(I_{M}, m+1\right)-U_{1}^{\delta}\left(I_{M}, m\right)\right) \leq \alpha_{m} u+\left(1-\alpha_{m}\right) d
$$

Let $\delta \in(0,1)$. Then it follows that

$$
\begin{aligned}
& \frac{1}{1-\delta}\left(U_{1}^{\delta}\left(I_{M}, m+1\right)-U_{1}^{\delta}\left(I_{M}, m\right)\right)= \\
& \sum_{t=1}^{\infty} d \delta^{t-1} \mu\left(B_{t}\right)+\sum_{t=1}^{\infty} u \delta^{t-1} \mu\left(A_{t}\right) \leq \\
& \sum_{t=1}^{\infty} d \mu\left(B_{t}\right)+\sum_{t=1}^{\infty} u \mu\left(A_{t}\right)= \\
& \alpha_{m} u+\left(1-\alpha_{m}\right) d .
\end{aligned}
$$

The following lemma was used above:

Lemma 2 Let $\left\{X_{n}\right\}_{n=1}^{\infty}$ be a sequence of independent and identically distributed random variables with mean equal to $\rho \leq \frac{1}{2}$ and finite variance $\sigma^{2}>0$ and let $c \in \mathbb{R}$. Then $\mu\left(\bigcap_{n=1}^{\infty}\left\{\boldsymbol{\omega}: \frac{S_{n}(\boldsymbol{\omega})}{n} \geq \frac{1}{2}-\frac{c}{n}\right\}\right)=0$.

Proof. The result will follow from the Law of Iterated Logarithm: Let $\left\{Y_{k}\right\}_{k=1}^{\infty}$ be independent and identically distributed random variables with $E\left[Y_{1}\right]=0$ and $\sigma^{2}\left(Y_{1}\right)=1$. Then $\varliminf_{n \rightarrow \infty} \frac{S_{n}}{\sqrt{2 n \log \log n}}=-1$ a.s. (Billingsley (1995), Theorem 9.5, page 154). 
Define $Y_{n}(\boldsymbol{\omega})=\frac{X_{n}(\boldsymbol{\omega})-\rho}{\sigma}$ and $S_{n}^{y}(\boldsymbol{\omega})=\sum_{k=1}^{n} Y_{k}(\boldsymbol{\omega})$. Then $\frac{S_{n}^{y}(\boldsymbol{\omega})}{n}=\frac{1}{\sigma}\left(\frac{S_{n}(\boldsymbol{\omega})}{n}-\right.$ $\rho)$. By the Law of Iterated Logarithm, there is $Z \subset \Omega$ with $\mu(Z)=0$ such that $\lim _{n \rightarrow \infty} \frac{S_{n}^{y}\left(\omega^{\infty}\right)}{\sqrt{2 n \log \log n}}=-1$, for all $\boldsymbol{\omega} \in \Omega \backslash Z$. Let $\boldsymbol{\omega} \in \Omega \backslash Z$. Then, since $\inf _{n \geq k} \frac{S_{n}^{y}(\boldsymbol{\omega})}{\sqrt{2 n \log \log n}}$ increases to $\varliminf_{n \rightarrow \infty} \frac{S_{n}^{y}(\boldsymbol{\omega})}{\sqrt{2 n \log \log n}}$, it follows that $\inf _{n \geq k} \frac{S_{n}^{y}(\boldsymbol{\omega})}{\sqrt{2 n \log \log n}} \leq-1$, for all $k$. That is, $\frac{S_{n}^{y}(\boldsymbol{\omega})}{\sqrt{2 n \log \log n}} \leq-1$ infinitely often. Thus, for $n$ large enough,

$$
\frac{S_{n}(\boldsymbol{\omega})}{n} \leq \rho-\frac{\sigma \sqrt{2 \log \log n}}{\sqrt{n}}<\frac{1}{2}-\frac{c}{n}
$$

infinitely often (the last inequality follows because for $n$ large enough $c<$ $\sigma \sqrt{2 n \log \log n} \rightarrow \infty)$. It follows then that $\boldsymbol{\omega} \notin \bigcap_{n=1}^{\infty}\left\{\boldsymbol{\omega}: \frac{S_{n}(\boldsymbol{\omega})}{n} \geq \frac{1}{2}-\frac{c}{n}\right\}$; hence $\bigcap_{n=1}^{\infty}\left\{\boldsymbol{\omega}: \frac{S_{n}(\boldsymbol{\omega})}{n} \geq \frac{1}{2}-\frac{c}{n}\right\} \subseteq Z$ and the result follows.

Lemma 3 There exists $\delta^{*} \in(0,1)$ such that for all $\delta \in\left(\delta^{*}, 1\right), I_{M}$ is subgame perfect.

Proof. By Proposition 3.11 of Mertens and Parthasarathy (1987)) (or Proposition 1 of Abreu (1988)) it is enough to show that no player can profitably deviate from $I_{M}$ by deviating just in the first stage. Again, because player 1's case is symmetric to player 2's, we deal only with the former.

It is clear that player 1 does not want to deviate from $B_{1}^{M}(M)=N P$, since by choosing $P$ when he has all the money he would reduce his utility today by $d$, and receive the same future utility. So we are left to show that he does not want to deviate from $B_{1}^{M}(m)$, for all $m=0, \ldots, M-1$.

Let $m \in\{0, \ldots, M-1\}$. If player 1 deviates from $B_{1}^{M}(m)$, and therefore chooses $N P$, his utility will be equal to

$$
\bar{U}:=(1-\delta) \delta U_{1}^{\delta}\left(I_{M}, m\right)
$$

while if he does not deviate, his utility will be equal to

$$
U_{1}^{\delta}\left(I_{M}, m\right)=(1-\delta)\left(-d+\delta U_{1}^{\delta}\left(I_{M}, m+1\right) .\right.
$$

Thus,

$$
\left.U_{1}^{\delta}\left(I_{M}, m\right)-\bar{U}=(1-\delta)\left[-d+\delta \frac{1}{1-\delta}\left(U_{1}^{\delta}\left(I_{M}, m+1\right)\right)-U_{1}^{\delta}\left(I_{M}, m\right)\right)\right] .
$$


By lemma 1,

$$
\begin{aligned}
& -d+\delta \frac{1}{1-\delta}\left(U_{1}^{\delta}\left(I_{M}, m+1\right)-U_{1}^{\delta}\left(I_{M}, m\right)\right) \underset{\delta \rightarrow 1}{\rightarrow} \\
& \underset{\delta \rightarrow 1}{\longrightarrow}-d+\alpha_{m} u+\left(1-\alpha_{m}\right) d>0 .
\end{aligned}
$$

Therefore, if we let $\delta^{*}$ be such that for all $\delta>\delta^{*}$

$$
-d+\delta \frac{1}{1-\delta}\left(U_{1}^{\delta}\left(I_{M}, m+1\right)-U_{1}^{\delta}\left(I_{M}, m\right)\right)>0
$$

for all $m \in\{0, \ldots, M-1\}$, then

$$
U_{1}^{\delta}\left(I_{M}, m\right)-\bar{U}>0
$$

for all $m \in\{0, \ldots, M-1\}$.

Lemma 4 Let $I \in \mathcal{A}_{N}$. Then,

$$
U_{i}(I)=\frac{1}{2|S|} \sum_{s \in S} \sum_{\omega \in \Omega} u_{i}(\omega, B(s)) .
$$

Proof. Let $I \in \mathcal{A}_{N}$, and let $\tilde{S}=\Omega \times S$. Then $I$ also induces a Markov chain $\tilde{\Pi}$ on $\tilde{S}$ satisfying

$$
\tilde{\pi}_{i, j}=\pi_{i_{2}, j_{2}} .
$$

Since $\Pi$ is symmetric and irreducible, then so will be $\tilde{\Pi}$. Hence, by Propositions 7.1, and 7.5 of Behrends (2000), we have that

$$
\lim _{n \rightarrow \infty} \tilde{\pi}_{i i}^{n r}=\frac{r}{|\tilde{S}|}=\frac{r}{2|S|}>0
$$

where $r$ is the period of $\tilde{\Pi}$. Thus, all states are positive, i.e., $\tilde{S}$ is a positive class.

So, by Theorem I.15.2 of Chung (1967), the sequence of functions

$$
\boldsymbol{\omega} \mapsto \frac{1}{n} \sum_{k=1}^{n} u_{i}\left(\omega_{k}, B\left(s\left(\omega_{k}\right)\right)\right)
$$

converges almost everywhere to

$$
\frac{1}{2|S|} \sum_{s \in S} \sum_{\omega \in \Omega} u_{i}(\omega, B(s)),
$$


and the result follows by the Lebesgue dominated converge theorem.

The following lemma states that in any subgame perfect equilibrium there has to be a "punishment" state, which is a seller refusing to produce for a buyer in our model.

Lemma 5 Let $I \in \mathcal{A}_{N}$. Then, for all $i=1,2$, there exists $s \in S$ such that $B_{i}(s)=N P$.

Proof. Suppose that for some $i \in\{1,2\}$, we have $B_{i}(s)=P$, for all $s \in S$. Let $\tilde{I}_{-i}$ be such that $B_{-i}(s)=N P$, for all $s \in S$. Then, we obtain $U_{-i}\left(I_{i}, \tilde{I}_{-i}\right)=u / 2$, while if $I_{-i} \neq \tilde{I}_{-i}$, then

$$
U_{-i}(I) \leq \frac{u}{2}-\frac{d}{2|S|}
$$

Since $I$ is a subgame perfect equilibrium, then $I_{-i}=\tilde{I}_{-i}$, and so $U_{i}(I)=$ $-d / 2$.

But, letting $\tilde{I}_{i}$ be such that $B_{i}(s)=N P$, for all $s \in S$, we obtain $U_{i}\left(\tilde{I}_{i}, I_{-i}\right)=U_{i}(\tilde{I})=0 \geq U_{i}(I)$. This shows that $I_{i}$ is not a best response to $I_{-i}$, which is a contradiction.

Proof of Theorem 1. Let $N \in \mathbb{N}$ be given, and let $M=N-1$. We first establish that $I_{M}$ belongs to $\mathcal{A}_{N}$. It is clear that $I_{M}$ is symmetric, and since, for all $m \in S_{M}$,

$$
\pi_{m M}^{(M)} \geq \underbrace{\pi_{m m+1} \cdots \pi_{M-1 M} \pi_{M M} \cdots \pi_{M M}}_{M-m \text { terms }}>0 .
$$

we obtain that for all $m, m^{\prime} \in S_{M}$,

$$
\pi_{m m^{\prime}}^{(2 M)} \geq \pi_{m M}^{(M)} \pi_{M m^{\prime}}^{(M)}>0
$$

that is, the Markov Chain induced by $I_{M}$ is irreducible.

Finally, to show that $I_{M}$ is a subgame perfect equilibrium, we use the following limit argument: Let $s \in S_{M}, I^{\prime}$, and $s^{\prime}$ be given. Then, by Lemma 6.1 of Secchi and Sudderth (2000) we obtain that

$U_{i}\left(I_{M}, s\right)=\lim _{\delta \uparrow 1} U_{i}^{\delta}\left(I_{M}, s\right) \geq \lim _{\delta \uparrow 1} U_{i}^{\delta}\left(\left(I_{i}^{\prime}, I_{-i}^{M}\right),\left(s^{\prime}, s_{-i}\right)\right)=U_{i}\left(\left(I_{i}^{\prime}, I_{-i}^{M}\right),\left(s^{\prime}, s_{-i}\right)\right)$.

Thus, $I_{M}$ is a subgame perfect equilibrium. 
We are left to show that $U_{1}\left(I_{M}\right)+U_{2}\left(I_{M}\right) \geq U_{1}(I)+U_{2}(I)$ for all $I \in \mathcal{A}_{N}$. For each $I \in \mathcal{A}_{N}$, recall that by Lemma 4 we have

$$
U_{i}(I)=\frac{1}{2|S|} \sum_{s \in S} \sum_{\omega \in \Omega} u_{i}(\omega, B(s)) .
$$

Let $S_{P}=\left\{s \in S: B_{2}(s)=P\right\}$ and $S_{N P}=\left\{s \in S: B_{2}(s)=N P\right\}$. By symmetry, $\left|S_{P}\right|=\left|\left\{s \in S: B_{1}(s)=P\right\}\right|$ and $\left|S_{N P}\right|=\left|\left\{s \in S: B_{1}(s)=N P\right\}\right|$. Then, letting

$$
f(s)=\left\{\begin{array}{c}
0 \text { if } B_{1}(s)=N P, \\
1 \text { otherwise }
\end{array}\right.
$$

we obtain,

$$
\begin{aligned}
U_{1}(I) & =\frac{1}{2|S|}\left[\left|S_{P}\right|(u-d f(s))-d\left|S_{N P}\right| f(s)-d\left|S_{P}\right|\right] \\
& \leq \frac{1}{2|S|}\left[\left|S_{P}\right|(u-d)\right] .
\end{aligned}
$$

Because $I$ is a subgame perfect equilibrium, $\left|S_{N P}\right| \geq 1$, and so $\left|S_{P}\right|=$ $|S|-\left|S_{N P}\right| \leq|S|-1$. Hence, it follows that,

$$
U_{1}(I) \leq \frac{u-d}{2}\left(1-\frac{1}{|S|}\right) \leq \frac{u-d}{2}\left(1-\frac{1}{M+1}\right)=U_{1}\left(I_{M}\right) .
$$

Since, by symmetry, $U_{2}\left(I_{M}\right)=U_{1}\left(I_{M}\right) \geq U_{1}(I)=U_{2}(I)$, the result follows.

\section{B A Monetary Automaton with Oscillating Prices}

In this appendix, we describe formally the monetary system in which the price level is 1 in odd periods, and 2 in even periods. We assume that there are $M$ units of money in the economy, with $M$ being an even natural number. Player 1 starts with $m_{0}$ of this $M$ units. The state space of $I_{M}^{2}$ is $S=\{0, \ldots, M\} \times\{e, o\}$, where $e$ stands for even, and $o$ for odd, and the initial state is $\bar{s}=\left(m_{0}, o\right)$. 
The transition function is given by is defined by:

$$
T(\omega, a,(m, \alpha))= \begin{cases}(m+1, e) & \text { if } \omega=1, a=P, m \leq M-1 \text { and } \alpha=o, \\ (m, e) & \text { if } \omega=1, a=P, m=M \text { and } \alpha=o, \\ (m+2, o) & \text { if } \omega=1, a=P, m \leq M-2 \text { and } \alpha=o, \\ (m, o) & \text { if } \omega=1, a=P, m \geq M-1 \text { and } \alpha=o, \\ (m-1, e) & \text { if } \omega=2, a=P, m \geq 1 \text { and } \alpha=o, \\ (m, e) & \text { if } \omega=2, a=P, m=0 \text { and } \alpha=o, \\ (m-2, o) & \text { if } \omega=2, a=P, m \geq 2 \text { and } \alpha=o, \\ (m, o) & \text { if } \omega=2, a=P, m \leq 1 \text { and } \alpha=o, \\ (m,-\alpha) & \text { otherwise, }\end{cases}
$$

where $-\alpha=e$ if $\alpha=o$, and $-\alpha=o$ if $\alpha=e$.

Finally, player 1's behavior function is defined as follows:

$$
B_{1}(m, \alpha)=\left\{\begin{array}{l}
P \text { if } m<M \text { and } \alpha=o \\
P \text { if } m<M-1 \text { and } \alpha=e \\
N P \text { otherwise }
\end{array}\right.
$$

and, similarly, for player 2:

$$
B_{2}(m)=\left\{\begin{array}{l}
P \text { if } m>0 \text { and } \alpha=o, \\
P \text { if } m>1 \text { and } \alpha=e, \\
N P \text { otherwise }
\end{array}\right.
$$

This automaton induces two Markov chains $\Pi^{o}$, and $\Pi^{e}$ on $\{0, \ldots, M\}$, the former describing the evolution of money holdings in odd periods, and the latter the same in even periods. One can easily see that both chains are symmetric and irreducible, and so we obtain (see Lemma 4, and its proof) that for $i=1,2$,

$$
\begin{aligned}
U_{i}\left(I_{M}^{2}\right) & =\frac{1}{2}\left(\frac{1}{2(M+1)} \sum_{m=0}^{M} \sum_{\omega=1}^{2} u_{i}(\omega, B(m, o))\right. \\
& \left.+\frac{1}{2(M+1)} \sum_{m=0}^{M} \sum_{\omega=1}^{2} u_{i}(\omega, B(m, e))\right) \\
& =\frac{u-d}{2}\left(1-\frac{3}{2(M+1)}\right)
\end{aligned}
$$




\section{References}

Abreu, D. (1988): "On the Theory of Infinitely Repeated Games with Discounting," Econometrica, 56, 383-396.

Aumann, R. (1981): "Survey of Repeated Games," in Essays in Game Theory and Mathematical Economics in Honor of Oskar Morgenstern. Bibliographisches Institut, Mannheim.

Behrends, E. (2000): Introduction to Markov Chains. Wieweg, Wiesbaden.

Billingsley, P. (1995): Probability and Measure. Wiley, New York.

Carmona, G. (2000): "Social Norms and Monetary Trading," mimeo.

Champ, B., And S. Freeman (1994): Modelling Monetary Economics. Wiley, New York.

Chung, K. (1967): Markov Chains. Springer-Verlag, New York.

DePree, J., and C. Swartz (1988): Introduction to Real Analysis. Wiley, New York.

Ellison, G. (1994): "Cooperation in the Prisoner's Dilemma with Anonymous Random Matching," Review of Economic Studies, 61, 567-588.

FudenberG, D., And E. Maskin (1986): "The Folk Theorem in Repeated Games with Discounting and Incomplete Information," Econometrica, 54, 533-554.

GatA, J. (1995): "Repeated Random Matching Games under Restricted Information," Ph.D. thesis, University of Minnesota.

Humphrey, D., L. P., and J. Vesala (1996): "Cash, Paper, and Electronic Payments : A Cross-Country Analysis," Journal of Money, Credit, and Banking, 28, 914-939.

Hurwicz, L. (1980): "Discussion," in Models of Monetary Economies, ed. by J. Kareken, and N. Wallace. Federal Reserve Bank of Minneapolis, Minneapolis.

Kalai, E., and W. Stanford (1988): "Finite Rationality and Interpersonal Complexity in Repeated Games," Econometrica, 56, 397-410. 
Kandori, M. (1992): "Social Norms and Community Enforcements," Review of Economic Studies, 59, 63-80.

Kiyotaki, N., and R. Wright (1989): "On Money as a Medium of Exchange," Journal of Political Economy, 97, 927-954.

Kocherlakota, N. (1998a): "Money is Memory," Journal of Economic Theory, 81, 232-251.

(1998b): "The Technological Role of Fiat Money," Federal Reserve Bank of Minneapolis Quarterly Review, 22(3), 2-10.

(2002): "The Two-Money Theorem," International Economic Review, forthcoming.

Kocherlakota, N., and N. Wallace (1998): "Incomplete RecordKeeping and Optimal Payment Arrangements," Journal of Economic Theory, 81, 272-289.

Mauss, M. (1990): The Gift: The Form and Reason of Exchange in Archaic Societies. Routledge, London.

Mertens, J.-F., and T. Parthasarathy (1987): "Equilibria for Discounted Stochastic Games," CORE Discussion Paper 8750.

Okuno-Fujiwara, M., and A. Postlewaite (1995): "Social Norms and Random Matching Games," Games and Economic Behavior, 9, 79-109.

Rubinstein, A. (1986): "Finite Automata Play the Repeated Prisoner's Dilemma," Journal of Economic Theory, 39, 83-96.

Samuelson, P. (1958): "An Exact Consumption-loan Model of Interest with or without the Social Contrivance of Money," Journal of Political Economy, 66, 467-482.

Secchi, P., And W. Sudderth (2000): "A Simple Two-Person Stochastic Game with Money," Dipartimento de Matematica, Politecnico di Milano.

Selten, R. (1975): "Reexamination of the Perfectness Concept for Equilibrium Points in Extensive Games," International Journal of Game Theory, $4,25-55$. 
Sobel, M. (1971): "Noncooperative Stochastic Games," Annals of Mathematical Statistics, 42, 1930-1935.

Townsend, R. (1986): "Economic Organization with Limited Communication," American Economic Review, 77, 954-971.

Wallace, N. (1997): "Absence-of-Double-Coincidence Models of Money: A Progress Report," Federal Reserve Bank of Minneapolis Quarterly Review, 21(1), 2-10.

(2001): "Whither Monetary Economics?," International Economic Review, 42(4), 847-869. 